\title{
Targeting HER2-Driven Cancers in Non-Breast Cancer Malignancies
} Simon P Langdon*

Division of Pathology, Institute of Genetics and Molecular Medicine, University of Edinburgh, Western General Hospital, Crewe Road South, Edinburgh EH4 2XU, UK

Recent results from several large breast cancer clinical trials have demonstrated the value of targeting the cell surface receptor HER2 (erbB2) with multiple HER2-targeted agents [1-3]. This includes the use of combined antibodies (trastuzumab plus pertuzumab) as first line therapy in metastatic breast cancer [1], combined antibody and small molecule tyrosine kinase inhibitor (trastuzumab plus lapatinib) in early breast cancer [2] and antibody-drug conjugate (trastuzumab-DM1) in resistant disease [3]. The success of these approaches may now, in turn, suggest consideration of the use of such strategies in other cancer types in which single agent HER2 targeted therapy has some activity but is considered too limited for current use.

The cell surface receptor HER2 (or ErbB2) is a member of the EGFR family that drives the growth of a significant percentage of breast cancers [4]. It is amplified in approximately $20 \%$ of breast cancers and outcome for patients with these tumors has been linked to poor survival [5]. Breast cancers are now routinely assayed for the presence of HER2 amplification and such cases frequently benefit from treatment with the humanised antibody trastuzumab (herceptin) or the small molecule tyrosine kinase inhibitor lapatinib which are targeted against the HER2 receptor [6]. Several strategies have now been developed to improve outcome in HER2 positive breast cancer and also overcome resistance to trastuzumab. Recently published trials have now confirmed that combinations of HER2 targeted drugs such as trastuzumab + pertuzumab [1] or trastuzumab + lapatinib [2] are more effective in HER2 breast cancers than the single agents. The use of the antibody-drug conjugate trastuzumab-DM1 which combines the activity of trastuzumab with targeted delivery of the anti-microtubule agent DM1, is also effective in trastuzumab-resistant disease [3].

While these strategies have been pioneered within breast cancer, there is increasing interest in the possibility that other tumor types with amplification or overexpression of HER2 may also be responsive to HER2-targeted therapies and benefit from these approaches. Cancer types such as gastric cancer, ovarian cancer and lung cancer can express high levels of HER2. The TOGA phase III clinical trial demonstrated improved survival when trastuzumab was added to chemotherapy versus chemotherapy alone in treatment of HER2-positive advanced gastric or gastro-oesophageal junction cancer [7]. Preclinical studies in ovarian and lung cancer xenografts have demonstrated that combined therapies of trastuzumab and pertuzumab are effective against xenograft models of these diseases [8,9]. Therefore, while trastuzumab has not had marked efficacy as a single agent in these less sensitive diseases, these new HER2 combination strategies may prove more clinically effective.

However, several considerations will need to be taken into account in extending these approaches to other cancer types. Since these other diseases generally have lower percentages of HER2 amplification, the level of antitumor activity obtained with HER2-directed strategies will need to be sufficiently beneficial to justify the costs of diagnostic testing to identify potentially responsive tumors. The ease of identification of these subgroups will also need to be considered and it may be that histological subgroups with known higher percentages of HER2 amplification should be investigated first. Furthermore, it would be valuable to identify additional biomarkers alongside HER2 that helps identify the sensitive population who are most likely to benefit. There are already biomarkers downstream of HER2 such as PTEN and SPROUTY $[10,11]$ which can help identify breast cancers that are likely to be more resistant to trastuzumab and these may have value in high HER2 expressing non-breast cancers to help predict sensitivity.

If the twin hurdles of sufficient antitumour efficacy and costeffectiveness of diagnostic testing can be overcome, then these useful HER2-targeted strategies may find wider applicability beyond just breast cancer and add to the growing use of targeted therapies in these other disease types.

\section{References}

1. Baselga J, Cortés J, Kim SB, Im SA, Hegg R, et al. (2012) CLEOPATRA Study Group. Pertuzumab plus trastuzumab plus docetaxel for metastatic breast cancer. N Engl J Med 366 : 109-119.

2. Baselga J, Bradbury I, Eidtmann H, Di Cosimo S, de Azambuja E, et al. (2012) on behalf of the NeoALTTO Study Team. Lapatinib with trastuzumab for HER2-positive early breast cancer (NeoALTTO): a randomised, open-label multicentre, phase 3 trial. Lancet 379: $633-640$.

3. Burris HA, Rugo HS, Vukelja SJ, Vogel CL, Borson RA, et al. (2011) Phase II Study of the Antibody Drug Conjugate Trastuzumab-DM1 for the Treatment of Human Epidermal Growth Factor Receptor 2 (HER2) -Positive Breast Cancer After Prior HER2-Directed Therapy. J Clin Oncol 29: 398-405

4. Hynes NE, Lane HA (2005) ERBB receptors and cancer: the complexity of targeted inhibitors. Nat Rev Cancer 5: 341-354.

5. Slamon DJ, Leyland-Jones B, Shak S, Fuchs H, Paton V, et al. (2001) Use of chemotherapy plus a monoclonal antibody against HER2 for metastatic breast cancer that overexpresses HER2. N Engl J Med 344: 783 -792.

6. Awada A, Bozovic-Spasojevic I, Chow L (2012) New therapies in HER2-positive breast cancer: a major step towards a cure of the disease. Cancer Treat Rev.

7. Bang YJ, Van Cutsem E, Feyereislova A, Chung HC, Shen L, et al. (2010) ToGA Trial Investigators. Trastuzumab in combination with chemotherapy versus chemotherapy alone for treatment of HER2-positive advanced gastric or gastro-oesophageal junction cancer (ToGA): a phase 3, open-label, randomised controlled trial. Lancet 376 : 687-697.

8. Scheuer W, Friess T, Burtscher H, Bossenmaier B, Endl J (2009) Strongly enhanced antitumor activity of trastuzumab and pertuzumab combination treatment on HER2-positive human xenograft tumor models. Cancer Res 69: 9330 - 9336.

9. Faratian D, Zweemer AJM, Nagumo Y, Sims AH, Muir M, et al. (2011) Trastuzumab and pertuzumab produce changes in morphology and estrogen receptor signalling in ovarian cancer xenografts revealing new treatment strategies. Clin Cancer Res 17: 4451- 4461.

*Corresponding author: Simon P Langdon, Breakthrough Breast Unit and Division of Pathology, Institute of Genetics and Molecular Medicine, University of Edinburgh, Western General Hospital, Crewe Road South, Edinburgh EH4 2XU, UK, E-mail: simon.langdon@ed.ac.uk

Received February 25, 2012; Accepted March 02, 2012; Published March 04 2012

Citation: Langdon SP (2012) Targeting HER2-Driven Cancers in Non-Breast Cancer Malignancies. J Mol Biomark Diagn 2:e105. doi:10.4172/21559929.1000e105

Copyright: () 2012 Langdon SP. This is an open-access article distributed under the terms of the Creative Commons Attribution License, which permits unrestricted use, distribution, and reproduction in any medium, provided the original author and source are credited 
Citation: Langdon SP (2012) Targeting HER2-Driven Cancers in Non-Breast Cancer Malignancies. J Mol Biomark Diagn 2:e105. doi:10.4172/21559929.1000e105

Page 2 of 2

10. Faratian D, Goltsov A, Lebedeva GMS, Mullen P (2009) Systems biology reveals new strategies for personalising cancer medicine and confirms PTEN's role in resistance to trastuzumab. Cancer Res 69: 6713 - 6720.
11. Faratian D, Sims AH, Mullen P, Kay C, Um I, et al. (2011) Sprouty 2 is an independent prognostic factor in breast cancer and may be useful for stratifying patients for trastuzumab therapy. PLos One 6: e23772. 